\title{
SPECTRAL EVOLUTION OF YOUNG STELLAR OBJECTS
}

\section{FRED C. ADAMS}

U. C. Berkeley, Berkeley, CA 94720

We suggest an evolutionary sequence, from protostars to pre-main-sequence stars, for the classification of young stellar objects. This sequence is derived by comparing the predictions of the theoretical models of Adams and Shu with the morphological classification scheme of Lada and Wilking. We first define the spectral index in the near- and mid-infrared, $n \equiv$ $d \log \left(\nu F_{\nu}\right) / d \log \nu$, and then interpret the class of sources with negative spectral indices as protostars. The inferred mass infall rates for these objects are generally consistent with the measured gas temperatures of $\sim 35 \mathrm{~K}$ in Ophiuchus, and of $\sim 10 \mathrm{~K}$ in Taurus. Fitting the data requires us to adopt cloud rotation rates in Ophiuchus which are typically an order of magnitude greater than in Taurus, and we speculate on the mechanistic origin for this difference. Next, we consider a subclass of $T$ Tauri stars with near- and mid-infrared excesses and positive or zero spectral indices. We find that the objects with the steeper indices can be understood as the post-infall products from the collapse of rotating cloud cores, where the infrared excesses arise from the simple reprocessing of visible stellar photons in optically thick but spatially thin disks. The sources with flatter spectra may require massive accretion disks. Given the existence of protostars and naked star/disk systems, there is a natural interpretation of another subclass of $\mathrm{T}$ Tauri stars, those with two peaks in their emergent spectral energy distributions. These are readily explained as intermediate cases in which dust envelopes, optically thin in the infrared, still surround the stars and disks, perhaps because of residual infall. Finally, we find that the theory can be extended to explain the spectral energy distribution of FU Orionis, a famous outburst source. Our model suggests that FU Orionis has a disk, but it offers no discrimination between the competing ideas that the outburst took place on the star or in the disk. 